\title{
Foreskin healing after distal hypospadias repair: Does stenting affect the outcome?
}

\author{
Ireneusz Honkisz ${ }^{1, A-D, F}$, Janusz Sulisławski1,B,E,F, Barbara Dobrowolska-Glazar ${ }^{1, B, E, F}$, \\ Caroline F. Kuijper ${ }^{2, A, E, F}$, Rafał Chrzan ${ }^{1, A, B, E, F}$ \\ 1 Department of Pediatric Urology, Jagiellonian University Medical College, Kraków, Poland \\ 2 Department of Pediatric Urology, Amsterdam University Medical Center, the Netherlands \\ A - research concept and design; $B$ - collection and/or assembly of data; $C$ - data analysis and interpretation; \\ $D$ - writing the article; $E$ - critical revision of the article; $F$ - final approval of the article
}

\section{Address for correspondence}

Rafał Chrzan

E-mail: rafal.chrzan@uj.edu.pl

Funding sources

None declared

Conflict of interest

None declared

\section{Acknowledgements}

We are grateful to Prof. Tom P.V.M. de Jong for his continuous stimulation and the critical feedback that helped improve the outcomes; to Mr. Mirosław Witkowski for the statistical support; and to Dr. M. Wolnicki who operated on some cases.

Received on May 23, 2020

Reviewed on June 7, 2020

Accepted on September 20, 2020
Cite as

Honkisz I, Sulisławski J, Dobrowolska-Glazar B, Kuijper CF, Chrzan R. Foreskin healing after distal hypospadias repair: Does stenting affect the outcome? Adv Clin Exp Med. 2020;29(12):1487-1490. doi:10.17219/acem/127677

DOI

10.17219/acem/127677

\section{Copyright}

Copyright by Author(s)

This is an article distributed under the terms of the

Creative Commons Attribution 3.0 Unported (CC BY 3.0)

(https://creativecommons.org/licenses/by/3.0/)

\begin{abstract}
Background. Distal penile hypospadias account for about $70 \%$ of all cases of hypospadias. There is a variety of operative techniques that could be performed when foreskin reconstruction is an option. The urethral stent is left in the urethra to prevent complications.

Objectives. To determine whether the duration of stenting influences the healing of foreskin after distal hypospadias repair.

Material and methods. Data from 2 institutions was retrospectively analyzed. Inclusion criteria were as follows: 1) a modified meatal advancement glanuloplasty without tubularization of the urethral plate, 2) foreskin reconstruction and 3) follow-up - 12 months. All other types of reconstruction and re-do procedures were excluded. The period of urethral stenting was determined intraoperatively depending on the surgeon's preferences. Mean age at operation was 23.3 months. The cohort was divided into 3 groups. In Group I (G-I), no catheter was left or it was removed the next day after surgery. In Group II, the catheter was left for more than 5 days. In those 2 groups, the surgery was done by different surgeons. Group III consisted of 35 patients who had a stent for $<2$ days, and the procedure was performed by the same surgeon. The $x^{2}$ with Yates's correction and Pearson's $x^{2}$ tests were used for the statistical analysis.
\end{abstract}

Results. Overall, 11 patients had foreskin dehiscence and needed re-do surgery. None of the patients required operation because of foreskin stenosis. Complications occurred in 3 out of 33 patients (9\%) in Group I, 2 out of 27 in Group II (7.4\%) and 6 out of 35 in Group III (17\%). There was no statistically significant difference between Groups I and II ( $p=0.8144)$, nor between Groups I and III ( $p=0.5344)$. In the non-parametric Pearson's $x^{2}$ test, no significant difference was found in such grouped data $(p=0.4239)$.

Conclusions. Prolonged urethral stenting does not reduce the risk of a re-do foreskin surgery after hypospadias repair.

Key words: complication, hypospadias, foreskin, preputioplasty, stenting 


\section{Introduction}

The meatal advancement and glanuloplasty procedure (MAGPI) and its modifications are commonly used for distal hypospadias repair (Fig. 1). Deep, lateral mobilization of the distal part of the urethra is done in our institution for distal/coronal hypospadias followed by its advancement and glanuloplasty. This procedure is called an advancedMAPGI (aMAGPI). With this method, suturing of the urethra is avoided, which might reduce the risk of such urethra-related complications as a fistula and/or dehiscence. Originally, MAGPI procedure was combined with circumcision. ${ }^{1}$ Preservation of the foreskin is an option that can be offered to the patient/caregivers, providing it is technically feasible (the foreskin is well developed for closure). Nevertheless, preputioplasty itself comes with some specific complications such as fistula/dehiscence of the foreskin or phimosis. ${ }^{2}$ The normal-looking meatus in a male is located slightly proximal to the tip of the glans. After hypospadias and foreskin repair, a slightly ventral position of the meatus results in deflection of the urinary stream towards the reconstructed prepuce. In order to allow for adequate wound healing and reduce the risk of complications, a catheter is often left in the bladder. In the past, the catheter was left for many days after any type of hypospadias correction. Currently, there is a common trend to perform hypospadias repair as a one-day procedure, with or without urinary diversion.

The goal of this study was to assess the impact of duration of urethra stenting on the healing of the foreskin after distal hypospadias repair using the aMAGPI procedure.

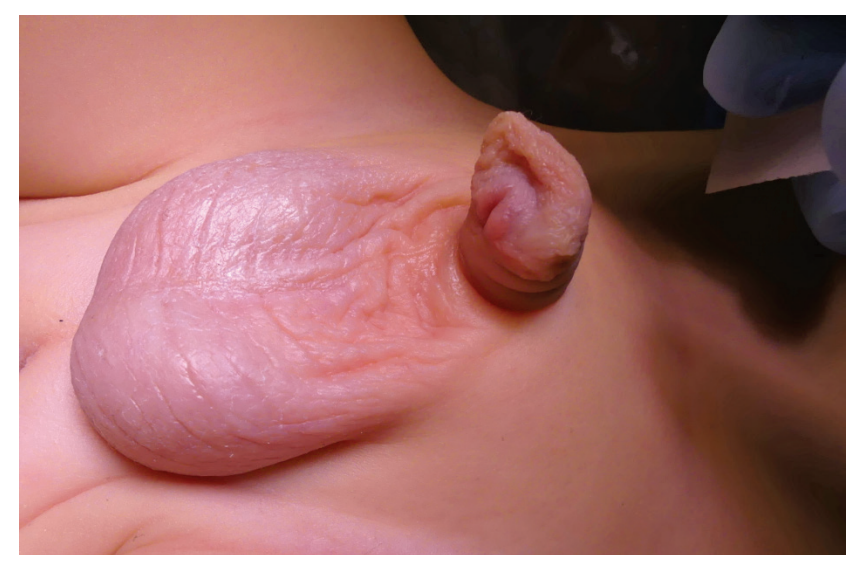

Fig. 1. Intraoperative view of the distal hypospadias and sufficient foreskin

\section{Material and methods}

A retrospective analysis of the distal hypospadias repairs performed at 2 institutions was undertaken. The inclusion criteria were: 1) a modified aMAGPI procedure, 2) foreskin reconstruction, 3) follow-up >12 months. All other types of reconstruction and the re-do procedures were excluded.
The duration of urethral stenting was determined intraoperatively depending on the surgeon's preferences.

At the University Children's Hospital in Kraków, Poland, the surgical corrections were performed by different surgeons. From October 2016, the duration of urethral stenting was significantly shortened to meet the international standards. The cohort from this institution was divided into 2 groups. In Group I (G-I), no catheter was left or it was removed the next day after surgery. In Group II (G-II), the catheter was left for more than 5 days. Group III (G-III) consisted of patients operated at the University Children's Hospital in Amsterdam, and the aMPGPI procedure with preputioplasty was performed by 1 surgeon (RC). In this group, no catheter was left or it was removed the next day after surgery.

\section{Surgical techniques}

A polypropylene traction suture is positioned through the glans. The transverse lip (bridge) of tissue distal to the meatus, if present, is divided by a longitudinal incision and the free edges of the wound are sutured together transversally. A catheter is then placed into the bladder.

A U-like incision is made around the meatus and then prolonged towards the top of the glans. The distal part (ca. 8-10 $\mathrm{mm}$ long) of the urethra is dissected free from the skin and corpora cavernosa and then advanced to the top of the glans. The glanular wings are approximated in the midline around the urethra by interrupted sutures $(5 / 0$ polyglactin). The ventral edge of the meatus is attached to the glans.

The major steps of foreskin reconstruction were the same in all cases. An U-like incision was made along the free margins of foreskin. The 2 layers were separated, and then the free edges were brought together with interrupted sutures (6/0 polyglactin) in 2 layers. A 10 Fr tube was left in the urethra and in the bladder (Fig. 2). In G-III, the dartos was approximated with 2-3 interrupted stiches whenever possible to identify and mobilize. Antibiotics (amoxicillin/clavulanic acid or cefuroxime depending on the local protocols) were administrated perioperatively.

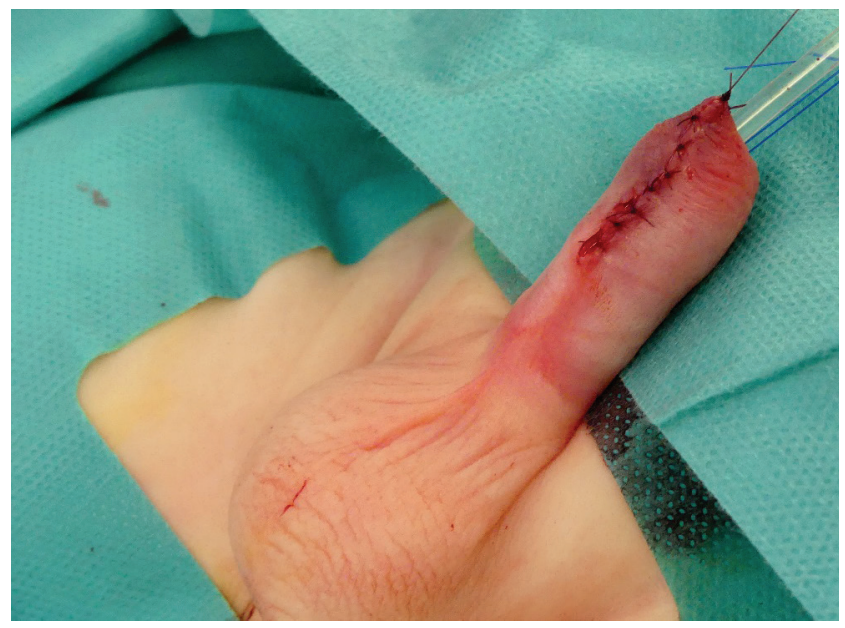

Fig. 2. Intraoperative view after foreskin reconstruction 


\section{Follow-up}

Each patient was assessed at the outpatient clinic between 6 and 12 months after the operation. Any distal foreskin dehiscence or a foreskin fistula requiring re-do surgery was defined as a failure.

Statistical analysis was performed using Dell Statistica, v. 13 (Dell Inc, Austin, USA). The $\chi^{2}$ test with Yates's correction was used to assess differences between G-I and G-II, G-I and G-III, as well as G-II and G-III. Additionally, Pearson's $X^{2}$ test was used to assess the relationship between the groups. A p-value $<0.05$ was considered significant.

\section{Results}

There were 33 patients aged from 5 to 81 months in G-I, 27 patients aged from 10 to 95 months in G-II and 35 patients aged from 4 to 22 months in G-III (Table 1). No intraoperative complications were encountered. In G-I, 3 of the 33 patients (9\%) developed foreskin dehiscence. In G-II, a catheter was kept for 5-9 days (mean time 7 days) and 2 out of 27 (7.4\%) had foreskin dehiscence. In G-III, 6 out of the 35 patients (17\%) developed foreskin dehiscence. The $\chi^{2}$ test with Yates's correction was used to check differences between G-I and G-II, as well as G-I and G-III. There was no statistically significant difference in the incidence of complications between Groups I and II ( $\mathrm{p}=0.8144)$, or between Groups I and III $(\mathrm{p}=0.5344)$. Also, in the non-parametric Pearson's $\chi^{2}$ test, the difference was not significant in such grouped data $(\mathrm{p}=0.4239)$.

\section{Discussion}

Distal penile hypospadias accounts for about $70 \%$ of all cases of hypospadias. There is a variety of operative techniques for distal hypospadias repairs, and most of them are combined with circumcision. However, in many cases, foreskin reconstruction can be offered as an option whenever technically possible. ${ }^{3-6}$

In this study, we focused only on the foreskin complications to find out whether the duration of stenting might affect the outcome. Many factors can influence the outcome of preputioplasty, such as suture material, the technique used, duration of stenting, as well as the experience of the surgeon. Under a few circumstances, the foreskin cannot be preserved, e.g., when needed for urethroplasty or when it is underdeveloped, making its reconstruction impossible.

The MAGPI procedure was devised by Duckett in 1981 for glandular hypospadias. ${ }^{1}$ When the meatus is located at the level of the corona, a simple MAGPI procedure might not be the optimal solution. Several other methods have been proposed for this type of hypospadias. Among the most commonly performed are the TIPU - tubularized incised urethral plate urethroplasty - and the Mathieu procedure. ${ }^{5-8}$ In our institution, a modified MAGPI procedure is used, which consists of an extensive mobilization of the urethral walls and its advancement.

Foreskin preservation was first described by Righini in 1961, and the principle of this procedure remained consistent. ${ }^{9}$ However, many surgeons still prefer circumcision because some prior studies showed an increase in complications of urethroplasty when combined with preputioplasty. ${ }^{4}$ In 1989, Frey and Cohen performed foreskin reconstruction in combination with a MAGPI procedure in 46 patients and experienced foreskin dehiscence in 10 patients $(21.7 \%){ }^{10}$

The reported foreskin related complication rate in the other types of hypospadias repair ranged from $2.5 \%$ to $34.8 \% .{ }^{11-13}$ In our cohort, $11.6 \%$ of the patients developed foreskin dehiscence requiring re-do surgery. No significant foreskin stenosis occurred in our study population.

Some recent studies demonstrated a satisfactory outcome of the prepuce without an increase in the rate of urethroplasty or skin complications in patients undergoing TIPU repair. 5,6,14 Moreover, it has been suggested that the reconstructed prepuce might act as a protective "layer" reducing the risk of the urethral fistulae. ${ }^{3}$

The prepuce reconstruction can be performed in 2 or 3 layers. The intermediate layer (dartos) is a thin connective tissue layer that can play an essential role in proper sliding between the inner and outer layers of the skin after reconstruction. ${ }^{12}$ In the majority of our cases, the repair was performed in 2 layers (6/0 polyglactin); however, in some patients, a few interrupted stiches were placed to approximate the dartos layer of the prepuce. This maneuver seems not to reduce the complication rate.

Also, suturing material has been considered to influence the outcome. Antao et al. reported an increased risk of foreskin-related complications (dehiscence and fistula) when the wound was closed in a running way (polyglactin rapid breakdown) in comparison to an interrupted manner (polyglactin). ${ }^{11}$ In our cohort, interrupted stitches were used (polyglactin).

Table 1. Patient data

\begin{tabular}{|c|c|c|c|}
\hline Group of patients & Catheterization & $\begin{array}{l}\text { Range of age (average, median) } \\
\text { in months }\end{array}$ & Foreskin dehiscence \\
\hline G-I - 33 patients (different surgeons) & 0 or 1 day & $5-81(22,16)$ & 3 patients (9\%) \\
\hline G-II - 27 patients (different surgeons) & $>5$ days & $10-95(36,29)$ & 2 patients $(7.4 \%)$ \\
\hline G-III - 35 patients (the same surgeon) & 0 or 1 day & $4-22(12,12)$ & 6 patients (17\%) \\
\hline
\end{tabular}


The role of the use of urethral stenting in distal hypospadias surgery is controversial, and usually, only the urethra-related complications are analyzed in this aspect. ${ }^{14-17}$ The foreskin is preferably used as an interposing layer to cover the urethroplasty. ${ }^{18,19}$ The impact of duration of stenting on the healing of the prepuce has not been studied yet.

We hypothesized that the urinary stream directed towards the reconstructed foreskin could increase the incidence of foreskin-related complication when there is no catheter left after reconstruction or when the catheter is removed shortly after the procedure. In the past, some surgeons have tended to leave the catheter for a long time even after distal hypospadias repair. Catheter placement has an impact on patients' postoperative comfort, which means it should be removed as soon as possible. In this study, different pediatric urologists were involved in the surgical treatment of the patients in G-I and G-II. The duration of stenting varied depending on the surgeon's preference, which might have affected the outcome. Group III was included in the analysis to minimalize the surgeon-related bias. The patients had been operated by the same pediatric urologist, and the catheter was removed shortly after the procedure. Although the complication rate in G-III was higher than in G-I and G-II, the difference was statistically insignificant.

\section{Limitations}

We realize that there are many different factors that might affect the final outcome of any surgical procedure. The inclusion criteria of our study were very strict to minimalize any bias. For this reason, the outcome of urethroplasty was not taken into consideration. The main concern was the number of surgeons performing surgery in G-I and G-II. The human-related bias was reduced by adding G-III analysis. The operating surgeons might likely have tended to leave a catheter a bit longer in older children, as the average age of patients in G-II was higher than in the other groups. No conclusion should be drawn from this as today the majority of patients are scheduled for this surgery between 6 and 18 months of life.

The small number of participants is another limitation of this study. Nevertheless, proper statistical methods have been chosen to compare the samples. Retrospective analysis has its limitations for the assessment of the outcome, but the endpoint of the study was clearly defined - partial (fistula) or total foreskin dehiscence requiring re-do surgery.

\section{Conclusions}

Prolonged urethral stenting does not reduce the risk of re-do foreskin surgery after hypospadias repair.

\section{ORCID iDs}

Ireneusz Honkisz (D) https://orcid.org/0000-0002-7448-9908 Janusz Sulisławski (1) https://orcid.org/0000-0002-4802-5210 Barbara Dobrowolska-Glazar (i) https://orcid.org/0000-0002-0192-9263 Caroline F. Kuijper (D) https://orcid.org/0000-0001-9668-5339 Rafał Chrzan (10) https://orcid.org/0000-0001-8620-1898

\section{References}

1. Duckett JW. MAGPI (meatoplasty and glanuloplasty): A procedure for subcoronal hypospadias. Urol Clin North Am. 1981;8(3):513-519.

2. Kallampallil J, Hennayake S. Foreskin retractility following hypospadias repair with preputioplasty: Medium term outcomes. J Pediatr Urol. 2013;9(6):1204-1209. doi:10.1016/j.jpurol.2013.05.022

3. Dewan PA. Distal hypospadias repair with preputial reconstruction. J Pediatr Child Health. 1993;29(3):183-184.

4. Suoub M, Dave S, El-Hout Y, Braga LH, Farhat WA. Distal hypospadias repair with or without foreskin reconstruction: A single-surgeon experience. J Pediatr Urol. 2008;4(5):377-380. doi:10.1016/j.jpurol. 2008.01.215

5. Snodgrass W, Dajusta D, Villanueva C, Bush N. Foreskin reconstruction does not increase urethroplasty or skin complications after distal TIP hypospadias repair. J Pediatr Urol. 2013;9(4):401-406. doi:10. 1016/j.jpurol.2012.06.008

6. Esposito C, Savanelli A, Escolino M, et al. Preputioplasty associated with urethroplasty for correction of distal hypospadias: A prospective study and proposition of a new objective scoring system for evaluation of esthetic and functional outcome. J Pediatr Urol. 2014;10(2):294-299. doi:10.1016/j.jpurol.2013.09.003

7. Subramaniam R, Spinoit AF, Hoebeke P. Hypospadias repair: An overview of the actual techniques. Semin Plast Surg. 2011;25(3):206-212. doi:10.1055/s-0031-1281490

8. El-Ganainy EO, Hameed DA, Abdelsalam YM, Abdelaziz MA. Prepuce preserving versus conventional Mathieu urethroplasty for distal hypospadias: A prospective randomized study. J Pediatr Urol. 2012; 8(3):264-267. doi:10.1016/j.jpurol.2011.05.004

9. Righini A. Symposium sur l'hypospadias. Ann Chir Infant. 1969;10:314.

10. Frey P, Cohen SJ. Reconstruction of foreskin in distal hypospadias repair. Prog Pediatr Surg. 1989;23:192-200.

11. Antao B, Lansdale N, Roberts J, Mackinnon E. Factors affecting the outcome of foreskin reconstruction in hypospadias surgery. J Pediatr Urol. 2007;3(2):127-131. doi:10.1016/j.jpurol.2006.06.003

12. Castagnetti M, Bagnara V, Rigamonti W, Cimador M, Esposito C. Preputial reconstruction in hypospadias repair. J Pediatr Urol. 2017;13(1): 102-109. doi:10.1016/j.jpurol.2016.07.018

13. Klijn AJ, Dik P, de Jong TP. Results of preputial reconstruction in 77 boys with distal hypospadias. J Urol. 2001;165(4):1255-1257.

14. Castagnetti M, Gnech M, Angelini L, Rigamonti W, Bagnara V, Esposito C. Does preputial reconstruction increase complication rate of hypospadias repair? 20-year systematic review and meta-analysis. Front Pediatr. 2016;28(4):41. doi:10.3389/fped.2016.00041

15. Buson H, Smiley D, Reinberg Y, Gonzales R. Distal hypospadias repair without stents: Is it better? J Urol. 1994;151(4):1059-1060.

16. Chalmers DJ, Siparsky GL, Wiedel CA, Wilcox DT. Distal hypospadias repair in infants without a postoperative stent. Pediatr Surg Int. 2015; 31(3):287-290. doi:10.1007/s00383-014-3647-y

17. Leclair MD, Camby C, Battisti S, Renaud G, Plattner V, Heloury Y. Unstented tubularized incised plate urethroplasty combined with foreskin reconstruction for distal hypospadias. Eur Urol. 2004;46(4): 526-530.

18. El-Karamany TM, Al-Adi AM, Omar RG, Abdel Aal AM, Eldakhakhny AS, Abdelbaki SA. A critical analysis of stented and unstented tubularized incised plate urethroplasty through a prospective randomised study and assessment of factors influencing the functional and cosmetic outcomes. Urology. 2017;107:202-208. doi:10.1016/j.urology. 2017.04.056

19. El-Kassaby AW, Al-Kandari AM, Elzayat T, Shoekeir AA. Modified tubularized incised plate urethroplasty for hypospadias repair: A longterm results of 764 patients. Urology. 2008;71(4):611-615. doi:10. 1016/j.urology.2007.11.121 